\title{
Doença granulomatosa crônica: diagnóstico no primeiro episódio infeccioso
}

\author{
Chronic granulomatous disease: diagnosis on the first infection episode
}

\begin{abstract}
Luís Roberto C. M. Bonilha1', Geraldo Roberto Cogo ${ }^{2}$, Antonio Condino Neto ${ }^{3}$, Edgar Borges de Oliveira Júnior ${ }^{4}$, José Vicente B. de Pieri ${ }^{5}$, César Carvalho Tonello ${ }^{5}$, Lourdes Josefina R. Cogo ${ }^{5}$, João Luiz D. Martins ${ }^{5}$, Luís Alberto Verri ${ }^{5}$
\end{abstract}

\section{RESUMO}

Objetivo: Relatar caso ilustrativo de doença granulomatosa crônica cujo diagnóstico ocorreu durante o aparecimento do primeiro episódio infeccioso, colaborando com a iniciativa do Brazilian Group for Immunodeficiency para a sensibilização do pediatra geral em relação ao diagnóstico precoce das imunodeficiências primárias, o que está associado a melhor qualidade de vida e maior sobrevida desses indivíduos.

Descrição do caso: Paciente do sexo masculino, 39 dias de vida, admitido em pronto-socorro pediátrico por febre alta há cinco dias e irritabilidade. No dia seguinte, observou-se abscesso cervical, isolando-se Staphylococcus aureus comunitário. Durante a internação, ocorreram outros abscessos superficiais e em cadeias ganglionares profundas, além de resposta lenta aos antimicrobianos. Solicitou-se investigação para imunodeficiências, que confirmou a hipótese de doença granulomatosa crônica por quantificação dos ânions superóxido e teste de redução do nitrobluetetrazolio. Paciente foi encaminhado a serviço especializado, no qual identificou-se doador de medula óssea compatível, realizando-se o transplante seis meses após o diagnóstico. Quatro meses após o transplante, ocorreu normalização do burst oxidativo, indicando sucesso.

Comentários: $\mathrm{O}$ paciente mostrou apresentação típica da doença, o que permitiu seu diagnóstico por pediatras gerais já na primeira infecção, tendo como consequência o acompanhamento por especialistas em imunodeficiências primárias,

Instituição: Hospital Vera Cruz, Campinas, SP, Brasil

${ }^{1}$ Mestre em Saúde da Criança e do Adolescente pela Universidade Estadual de Campinas (Unicamp); Médico do Hospital Vera Cruz, Campinas, $\mathrm{SP}$, Brasil

${ }^{2}$ Chefe do Departamento de Pediatria do Hospital Vera Cruz, Campinas, SP, Brasil

${ }^{3}$ Professor Associado e Livre-docente do Departamento de Imunologia do Instituto de Ciências Biomédicas da Universidade de São Paulo (USP), São Paulo, SP, Brasil

${ }^{4}$ Doutorando em Imunologia pelo Instituto de Ciências Biológicas da USP, São Paulo, SP, Brasil

${ }^{5}$ Médico do Hospital Vera Cruz, Campinas, SP, Brasil a introdução da profilaxia antimicrobiana e a procura bem sucedida de doador de medula HLA-compatível.

Palavras-chave: doença granulomatosa crônica; síndromes de imunodeficiência; abscesso.

\section{ABSTRACT}

Objective: To report a case of chronic granulomatous disease diagnosed during the first infectious episode in order to collaborate with the Brazilian Group for Immunodeficiency, in sensitizing the general pediatrician that the early diagnosis of primary immunodeficiency results in better quality of life and longer life expectancy for the patients.

Case Description: Male patient, 39 days, admitted to the pediatric emergency ward with fever for the last five days and irritability. On the following day, a cervical abscess was noted and a community Staphylococcus aureus was isolated. During hospital stay, other abscesses were observed in the skin and in the deep ganglia chains, with a slow response to antibiotics. Investigation of immunodeficiency was requested and chronic granulomatous disease was confirmed by quantification of superoxide anions and nitrobluetetrazolium tests. The patient was transferred to a specialized clinic for bone marrow transplantation, performed six months after diagnosis. Four months afterwards, the normalization of oxidative burst was noted, indecating the success of the transplantation.
Endereço para correspondência:

Luís Roberto de Castro Martins Bonilha

Avenida Dr. Nelson Noronha Gustavo Filho, 150, apto. 24-B - Vila Brandina CEP 13092-526 - Campinas/SP

E-mail: luis.bonilha@hotmail.com

Conflito de interesse: nada a declarar

Recebido em: 6/5/2009

Aprovado em: 30/11/2009 
Comments: The patient showed a typical presentation of the disease, which allowed its diagnosis by general pediatricians on the first infection, allowing the follow-up by experts in primary immunodeficiencies, the introduction of antimicrobial chemoprophylaxis, and the successful search for an HLA-compatible bone marrow donor.

Key-words: granulomatous disease, chronic; immunologic deficiency syndromes; abscess.

\section{Introdução}

A doença granulomatosa crônica (DGC) é uma imunodeficiência primária incomum (afeta cerca de 1/250.000 nascidos vivos), causada por um defeito na produção de intermediários no metabolismo do oxigênio nos fagócitos, que interferem na sua capacidade de destruir micro-organismos catalase positivos e intracelulares ${ }^{(1)}$.

A forma mais comum da doença é ligada ao cromossomo $\mathrm{X}$ e deve-se a uma alteração na gp91-phox ${ }^{(2)}$. Manifesta-se usualmente nos primeiros meses de vida, com infecções de repetição, sendo as mais comuns: abscessos, linfadenites, pneumonias e otites ${ }^{(1,3-6)}$. Também existem as formas autossômicas recessivas, em que as manifestações são geralmente mais tardias, menos graves e com menor morbimortalidade ${ }^{(2,4,7)}$.

O diagnóstico da DGC se dá pelo histórico de infecções graves e recorrentes, de início precoce, geralmente afetando as barreiras do organismo e a demonstração bioquímica de defeito na explosão oxidativa dos fagócitos, por meio do teste de redução do nitrobluetetrazolio (NBT), dosagem da produção do ânion superóxido ou pela citometria de fluxo, usando-se o método da dihidrorhodamina (DHR) $)^{(8)}$.

Até o presente, a cura para a DGC é obtida apenas pelo transplante de medula com doador HLA-compatível, que deve ser feito preferencialmente antes da adolescência, quando a mortalidade aumenta ${ }^{(9)}$. Para os que não têm doador compatível, o transplante com células-tronco do cordão umbilical está em fase experimental, com relatos de sucessos $^{(10-12)}$.

Nas últimas décadas, a profilaxia antimicrobiana, usualmente com trimetoprima-sulfametoxazol e itraconazol, reduziu significativamente a incidência de infecções e suas complicações, melhorando a sobrevida ${ }^{(8,13,14)}$. Além da profilaxia, o seguimento preventivo periódico desses pacientes, desde o diagnóstico, também parece aumentar a sua sobrevi$\mathrm{da}^{(15)}$. A utilização do interferon gama (IFN- $\gamma$ ) não ocorre em todos os $\operatorname{casos}^{(16)} \mathrm{e}$ ainda há controvérsias na literatura sobre as evidências de benefícios para seu uso profilático ${ }^{(1,14,17,18)}$. No seguimento preventivo desses pacientes, é necessário rastrear a presença de coriorretinite, anormalidade frequentemente associada à DGC ${ }^{(19,20)}$.

No Brasil, relatos de casos publicados ${ }^{(2,8,21,22)}$ mostram crianças com diagnóstico realizado entre cinco e dez anos de vida. Casuísticas maiores mostram que a forma mais comum, ligada ao cromossomo $\mathrm{X}$, tem seu diagnóstico realizado ao redor dos três anos, em média dois anos após o aparecimento da primeira infecção $0^{(4,9,13,23)}$.

Nesse contexto, o objetivo do presente estudo foi relatar caso ilustrativo de DGC, cujo diagnóstico ocorreu durante o aparecimento do primeiro episódio infeccioso, colaborando com a iniciativa do Brazilian Group for Immunodeficiency para a sensibilização do pediatra geral em relação ao diagnóstico precoce das imunodeficiências primárias, o que está associado a melhor qualidade de vida e maior sobrevida desses indivíduos.

\section{Descrição do caso}

Paciente do sexo masculino, nascido a termo com peso de $3550 \mathrm{~g}$ e Boletim de Apgar com $1^{\circ}$ e $5^{\circ}$ minutos de 9 e 9 . A criança não apresentava antecedentes familiares de imunodeficiências ou óbitos em crianças. $\mathrm{O}$ paciente recebeu alta com 48 horas de vida em aleitamento materno exclusivo.

Com 39 dias de vida, foi admitido no serviço de prontosocorro infantil com história de febre alta $\left(\right.$ até $\left.40^{\circ} \mathrm{C}\right)$ e irritabilidade há cinco dias, chegando a três picos por dia, porém aceitando bem o seio materno, sem diarreia ou vômitos. Além da irritabilidade, não apresentava outras alterações no exame físico. Foi, então, feito hemograma, líquor e exame do sedimento urinário. O líquor estava límpido e incolor, com 4,0 células/uL, 2,0 hemácias/uL, 59mg/dL de glicose, 38mg/ dL de proteínas e reação de Pandy negativa. O hemograma mostrava hemoglobina de 9,6g/dL, hematócrito de 29,6\%, 16.600 leucócitos $/ \mathrm{mm}^{3}$, com $5 \%$ bastonetes, $47 \%$ segmentados, 33\% linfócitos, $1 \%$ monócitos e 370.000 plaquetas/ $\mathrm{mm}^{3}$. No exame de urina, havia 2.400 leucócitos $/ \mathrm{mL}$ e 30.000 hemácias $/ \mathrm{mL}$. A urocultura não mostrou crescimento bacteriano. Na hemocultura, detectou-se Staphylococcus epidermidis, interpretado como contaminação pela equipe de Microbiologia. O paciente foi, então, internado com diagnóstico de febre de etiologia indeterminada em lactente jovem e iniciada ceftriaxona empírica, baseada na idade, na febre confirmada e na irritabilidade. Com um dia de internação, observou-se tumoração ganglionar em região cervical, mais evidente à direita, 
acompanhada de secreção ocular de aspecto amarelado. Nesse dia, novo hemograma apresentou piora da leucocitose (19.800/ $\mathrm{mm}^{3}$ ) e do desvio à esquerda, com $28 \%$ bastonetes. Foram colhidas sorologias (HIV, citomegalovírus, toxoplasmose, rubéola, sífilis, herpes simples e Epstein Barr) para investigação

Quadro 1 - Investigação sérica

\begin{tabular}{|lcc|}
\hline Exame & Paciente & Valor de referência \\
\hline \multicolumn{4}{|l}{ Eletroforese de proteínas $(\mathrm{g} / \mathrm{dL})$} \\
Proteínas totais & 6,8 & $6,40-8,20$ \\
Albumina & 3,69 & $3,20-5,00$ \\
Alfa 1 globulina & 0,32 & $0,10-0,40$ \\
Alfa 2 globulina & 0,94 & $0,40-0,90$ \\
Beta globulina & 0,76 & $0,60-1,00$ \\
Gama globulia & 1,09 & $0,70-1,50$ \\
Dosagem de imunoglobulinas (mg/dL) & \\
IgG & 933 & $270-780$ \\
IgM & 187 & $12-87$ \\
IgA & 39 & $6-58$ \\
Dosagem de complemento (mg/dL) & \\
CH50** & 193 & $\geq 60$ \\
C3 & 176 & $90-180$ \\
C4 & 55 & $10-40$ \\
\hline
\end{tabular}

* 1-3 meses; **u/CAE. de adenomegalia. Todas as sorologias mostraram-se negativas no decorrer da internação.

No segundo dia de internação, como surgiu flutuação da massa cervical, realizou-se biópsia de cadeia ganglionar cervical guiada por ultrassom. Aspirou-se material purulento do interior do tecido linfático, no qual isolou-se Staphylococcus aureus comunitário, sensível a todos os antimicrobianos testados. Associou-se oxacilina à ceftriaxona. Por meio da ressonância nuclear magnética de regiões cervical, torácica e abdominal foi possível detectar múltiplas linfonodomegalias de aspecto coalescente e com centro necrótico, acometendo as cadeias ganglionares cervical, mediastinal, retroperitoneal e inguinal direita. Com três dias de evolução, já em uso de ceftriaxona e oxacilina, ocorreu a formação de um abscesso perianal, em uma região que já apresentava lesões sugestivas de candidíase cutânea. Apenas após 28 dias de oxacilina e 21 dias de ceftrixona, ocorreu a completa resolução do quadro. Com 21 dias de internação, realizou-se investigação para imunodeficiências, e os resultados podem ser visualizados nos Quadros 1 e 2. Os resultados foram compatíveis com DGC.

Após o diagnóstico, o paciente foi prontamente encaminhado para acompanhamento especializado, com introdução imediata de quimioprofilaxia com trimetoprima-sulfametoxazol e itraconazol. Iniciou-se, então, a pesquisa de doador de medula óssea, que foi bem sucedida (irmã). A realização

Quadro 2 - Investigação celular

\begin{tabular}{|c|c|c|c|c|}
\hline \multirow{2}{*}{$\begin{array}{l}\text { Exame } \\
\text { Subpopulação linfocitária* }\end{array}$} & \multicolumn{2}{|c|}{ Paciente } & \multicolumn{2}{|c|}{ Valor de referência } \\
\hline & & & & \\
\hline Linfócitos CD3 & $74 \%$ & $2494 \mathrm{~mm}^{3}$ & $55-82 \%$ & $3500-5000 \mathrm{~mm}^{3}$ \\
\hline Linfócitos CD4 & $45,4 \%$ & $1526 \mathrm{~mm}^{3}$ & $50-57 \%$ & $2800-3900 \mathrm{~mm}^{3}$ \\
\hline Linfócitos CD8 & $24,6 \%$ & $827 \mathrm{~mm}^{3}$ & $8-31 \%$ & $350-2500 \mathrm{~mm}^{3}$ \\
\hline Relação CD4/CD8** & 1,85 & & $1,17-6,22$ & \\
\hline Nihobluetetrazolio*** & & & $\leq 5$ (SE) & $\geq 95$ (PMA) \\
\hline Paciente & 0 (SE) & 0 (PMA) & & \\
\hline Mãe & 0 (SE) & 100 (PMA) & & \\
\hline Pai & 0 (SE) & 100 (PMA) & & \\
\hline \multicolumn{5}{|l|}{ Ânion superóxido } \\
\hline Paciente & $0,77(\mathrm{SE})$ & 0,2 (PMA) & & \\
\hline Mãe & 0,27 (SE) & 6,27 (PMA) & & \\
\hline \multicolumn{5}{|l|}{ Polimorfonucleares } \\
\hline Paciente & 0,50 (SE) & 0,85 (PMA) & & \\
\hline Mãe & 1,350 (SE) & 9,9 (PMA) & & \\
\hline
\end{tabular}

${ }^{*} 0-6$ meses; ${ }^{* *} 0-36$ meses; ${ }^{* * *} \%$ células positivas; ${ }^{* * *}$ nmoles de $\mathrm{O}_{2} / 10^{6}$ células/60 minutos; SE: sem estímulo; PMA: pós-estímulo com PMA 
do transplante ocorreu seis meses após o diagnóstico e a normalização do burst oxidativo ocorreu quatro meses após o procedimento.

\section{Comentários}

O paciente aqui descrito mostrou quadro clínico esperado para a forma mais comum da DGC, ligada ao cromossomo X, com aparecimento de abscessos e linfadenites por micro-organismos catalase positivos, no caso, o Staphylococcus aureus ${ }^{(1,3-6)}$. Apesar disso, por se tratar de paciente com primeiro quadro infeccioso, em que não havia antecedente familiar de imunodeficiências ou óbitos em crianças, a hipótese inicial (após o aparecimento da linfonodomegalia e a aspiração do seu conteúdo) foi apenas de adenite bacteriana por Staphylococcus aureus comunitário (multissensível). Entretanto, a literatura mostra que esses casos de adenite bacteriana ocorrem usualmente diante de prematuridade com hospitalização prolongada ou em surtos epidêmicos em berçários ou UTI neonatal por Staphylococcus aureus multirresistente. Os fatores que modificaram a forma como a equipe entendia o caso e motivaram a solicitação do NBT foram a idade incomum para adenite bacteriana, a infecção por Staphylococcus aureus comunitário sem porta de entrada e a presença de tumoração em cadeia cervical e em cadeias profundas.

Ressalta-se aqui o diagnóstico precoce, que evitou sequelas e sofrimento, motivando a busca imediata de serviço especializado e o encontro de um doador compatível, levando o paciente à cura da sua doença ${ }^{(2,4,8,9,13,21,22)}$. O diagnóstico precoce permitiu o seguimento com especialistas, a introdução de quimioprofilaxia com trimetoprima-sulfametoxazol e itraconazol, a pesquisa de doador de medula óssea bem sucedida (irmã) e a realização do transplante seis meses após o diagnóstico, com normalização do burst oxidativo, quatro meses após o procedimento. Mesmo

\section{Referências bibliográficas}

1. Soler-Palacín $P$, Margareto $C$, Llobet $P$, Asensio $O$, Hernández $M$, Caragol I et al. Chronic granulomatous disease in pediatric patients: 25 years of experience. Allergol Immunopathol (Madr) 2007:35:83-9.

2. Prando-Andrade C, Agudelo-Florez P, Lopez JA, Paiva MA, Costa-Carvalho BT, Condino-Neto A. Autosomal chronic granulomatous disease: case report and mutation analysis of two Brazilian siblings. J Pediatr (Rio J) 2004;80:425-8. quando não ocorre o transplante por falta de doador, esse acompanhamento e a quimioprofilaxia fazem com que o diagnóstico precoce aumente a sobrevida e a qualidade de vida, com menos infecções e complicações, como a coriorretinite $^{(8,13-15,19,20)}$.

No Brasil e no mundo, apesar de vários casos diagnosticados em idade até menor que a desse paciente ${ }^{(1,4,6,7,13,14,17)}$, esse diagnóstico precoce é induzido pela investigação, em irmãos ou parentes de primeiro grau, de casos diagnosticados anteriormente por infecções de repetição. No Brasil, país em que existem iniciativas para que os pediatras recebam informação sobre o tema ${ }^{(23)}$, o diagnóstico ocorre em média dois anos após a primeira infecção $0^{(4,9,13,22)}$. Esse hiato pode permitir o aparecimento de infecções graves que resultam em morte ou sequelas.

O transplante de medula óssea teve início já na década de 1950, sendo os primeiros pacientes transplantados para o tratamento de doenças hematológicas. Quase 20 anos depois, o transplante passou a ser feito também para o tratamento de imunodefociências primárias, incluindo a DGC. A análise de 27 transplantes de medula óssea em pacientes com DGC, entre 1985 e 2000, evidenciou sobrevida de 23 casos e cura de $22^{(10)}$. A ausência de infecção no momento do transplante parece ser o principal indicador de bom prognóstico. No Brasil, a experiência com o transplante de medula óssea para imunodeficiências primárias ainda é incipiente e não há publicação formal sobre o tema.

Concluindo, a DGC é uma imunodeficiência primária incomum (afeta cerca de 1/250.000 nascidos vivos). Suspeitar que ela possa ser a doença de base em uma infecção por um micro-organismo catalase positivo sem uma razão aparente e/ou com uma evolução fora do esperado pode conduzir o pediatra ao diagnóstico precoce e à tomada de medidas que, se não levarem à cura por meio do transplante de medula óssea, poderão reduzir a chance de novas infecções graves, com menor morbimortalidade.
3. Grumach AS, Bellinai-Pires R, Araujo IS, Gonzalez CH, Carneiro-Sampaio MM. Chronic granulomatous disease of childhood: differential diagnosis and prognosis. Rev Paul Med 1993;111:472-6.

4. Winkelstein JA, Marino MC, Johnston RB Jr, Boyle J, Curnutte J, Gallin JI et al. Chronic granulomatous disease. Report on a national registry of 368 patients. Medicine (Baltimore) 2000;79:155-69. 
5. Khanna G, Kao SC, Kirby P, Sato Y. Imaging of chronic granulomatous disease in children. Radiographics 2005;25:1183-95.

6. Liese J, Kloos S, Jendrossek V, Petropoulou T, Wintergerst U, Notheis G et al. Long-term follow-up and outcome of 39 patients with chronic granulomatous disease. J Pediatr 2000;137:687-93.

7. Holland SM. Chronic granulomatous disease. Clin Rev Allergy Immunol 2010;38:3-10.

8. Moreira RJ, Barreto ICP, Daguer MGF, Brito NMB, Barreto BAP. Doença granulomatosa crônica: relato de caso. Rev Bras Alergia Imunopatol 2005;28:315-21.

9. Pasic S, Minic A, Minic P, Veljkovic D, Lilic D, Slavkovic B et al. Long-term follow-up and prognosis of chronic granulomatous disease in Yugoslavia: is there a role for early bone marrow transplantation? J Clin Immunol 2003;23:55-61.

10. Seger RA. Modern management of chronic granulomatous disease. $\mathrm{Br} \mathrm{J}$ Haematol 2008;140:255-66.

11. Parikh SH, Szabolcs P, Prasad VK, Lakshminarayanan S, Martin PL, Driscoll TA et al. Correction of chronic granulomatous disease after second unrelated-donor umbilical cord blood transplantation. Pediatr Blood Cancer 2007;49:982-4

12. Mochizuki K, Kikuta A, Ito M, Akaihata M, Sano H, Ohto H et al. Successful unrelated cord blood transplantation for chronic granulomatous disease: a case report and review of the literature. Pediatr Transplant 2009;13: 384-9.

13. Cale CM, Jones AM, Goldblatt D. Follow up of patients with chronic granulomatous disease diagnosed since 1990. Clin Exp Immunol 2000;120:351-5.

14. Martire B, Rondelli R, Soresina A, Pignata C, Broccoletti T, Finocchi A et al. Clinical features, long-term follow-up and outcome of a large cohort of patients with Chronic Granulomatous Disease: an Italian multicenter study. Clin Immunol 2008;126:155-64.

15. Roesler J, Koch A, Pörksen G, von Bernuth H, Brenner S, Hahn G et al. Benefit assessment of preventive medical check-ups in patients suffering from chronic granulomatous disease (CGD). J Eval Clin Pract 2005;11:513-21.

16. Carnide EG, Jacob CA, Castro AM, Pastorino AC. Clinical and laboratory aspects of chronic granulomatous disease in description of eighteen patients. Pediatr Allergy Immunol 2005;16:5-9.

17. Jones LB, McGrogan P, Flood TJ, Gennery AR, Morton L, Thrasher A et al Special article: chronic granulomatous disease in the United Kingdon and Ireland: a comprehensive national patient-based registry. Clin Exp Immunol 2008;152:211-8.

18. [No authors listed]. A controlled trial of interferon gamma to prevent infection in chronic granulomatous disease. The International Chronic Granulomatous Disease Cooperative Study Group. N Engl J Med 1991;324:509-16.

19. Goldblatt D, Butcher J, Thrasher AJ, Russell-Eggitt I. Chorioretinal lesions in patients and carriers of chronic granulomatous disease. J Pediatr 1999;134:780-3

20. Kim SJ, Kim JG, Yu YS. Chorioretinal lesions in patients with chronic granulomatous disease. Retina 2003;23:360-5.

21. Cardieri JMA, Nakaie CMA, Rozov T. Doença granulomatosa crônica da infância: relato de um caso. Pediatria (São Paulo) 1985;7:217-21.

22. Agudelo-Flórez P, Prando-Andrade CC, López JA, Costa-Carvalho BT, Quezada A, Espinosa FJ et al. Chronic granulomatous disease in Latin American patients: clinical spectrum and molecular genetics. Pediatr Blood Cancer 2006;46:243-52.

23. BRAGID. Brazilian Group for Immunodeficiency [homepage on the Internet] Imunodeficiência primária: os 10 sinais de alerta [cited 2008 Jun 6]. Available from: www.imunopediatria.org.br 